\title{
Vinorelbine-induced regression of a choroidal metastasis from primary breast carcinoma
}

\author{
Malvika Arya and Jay S. Duker*
}

\begin{abstract}
Background: Various therapeutic options exist to treat choroidal metastatic lesions. However, they are all associated with potential long-term adverse effects. This case report discusses a case of choroidal metastasis from primary breast carcinoma that regressed after single-agent chemotherapy.
\end{abstract}

Case presentation: We report a case of choroidal metastasis from estrogen receptor (ER) positive breast carcinoma that became resistant to endocrine therapy. The primary malignancy was treated with surgical resection and adjuvant chemoradiation, followed by hormone therapy with various agents in combination with kinase inhibitors for ER resistance. The choroidal metastatic lesion regressed after the initiation of vinorelbine. Vinorelbine is a cytotoxic vinca alkaloid with tolerable systemic adverse effects.

Conclusions: This case report highlights the possible role of vinorelbine as a single chemotherapeutic agent for the conservative therapy of uveal metastasis from advanced breast carcinoma, irrespective of responsiveness to hormone therapy.

Keywords: Choroidal neoplasm, Uveal neoplasm, Breast cancer, Vinorelbine, Antineoplastic agents phytogenic, Chemotherapeutic anticancer agents, Optical coherence tomography, OCT, Choroid

\section{Background}

The choroid is the primary ocular site for metastatic cancer due to its rich vascular supply and fenestrated choriocapillaris [1-3]. In women, the breast is the predominant site of primary neoplasms, and choroidal metastatic lesions appear in approximately $8 \%$ of patients with breast carcinoma [3]. Choroidal metastatic lesions secondary to breast cancer are often bilateral and located close to the posterior pole [1]. Uveal metastatic lesions may appear during systemic dissemination and are associated with a limited life expectancy $[1,4]$.

$60-70 \%$ of breast carcinomas are estrogen receptor (ER) positive and are responsive to endocrine therapy [5-7]. These tumors are treated with Tamoxifen in pre-menopausal women and aromatase inhibitors in

*Correspondence: jduker@tuftsmedicalcenter.org New England Eye Center, Tufts Medical Center, 800 Washington Street, Box 450, Boston, MA 02116, USA post-menopausal women, often after surgical resection of the primary lesion. However, a persistent risk of tumor recurrence remains, either from loss of ER expression or from resistance to hormone therapy by a mutation in the ER pathway [8]. This study reports a case who developed a choroidal metastatic lesion, while on therapy with selective estrogen receptor modulators (SERMs) for ER positive breast carcinoma, which then regressed following systemic chemotherapy with vinorelbine.

\section{Case presentation}

A 58-year-old female presented to New England Eye Center in June 2017 with decreased vision in her left eye of approximately 2 weeks duration. Her past ophthalmic history was significant for a retrobulbar migraine in her left eye. On presentation, her best-corrected visual acuity was $20 / 20$ in the right eye, which stayed consistent throughout her follow-up visits, and 20/40 in the left eye. Funduscopic exam of the affected eye revealed a $5.8 \mathrm{~mm}$ 
in diameter, yellow-colored choroidal mass located superior and temporal to the macula, as shown in Fig. 1a. Optical coherence tomography (OCT) and ultrasound of the corresponding site revealed subretinal fluid associated with a $2.47 \mathrm{~mm}$ choroidal lesion with medium internal reflectivity (Fig. 1b, c). Fundus autofluorescence of the lesion also revealed a hyper-autofluorescent choroidal mass with a surrounding pocket of subretinal fluid (Fig. 1d). Imaging of the right eye was within normal limits.

Her medical history was significant for stage IIIA T3 N1 M0, ER positive, progesterone receptor (PR) positive, human epidermal growth factor receptor 2 (HER2) negative, well-differentiated invasive ductal carcinoma of the right breast. A tumor measuring $6 \mathrm{~cm}$ was first diagnosed by screening mammogram 16 years prior to ocular presentation, in May 2001. She subsequently underwent a modified radical mastectomy of the right breast with sentinel and axillary lymph node dissection in June 2001. Surgical margins were free of the tumor. One sentinel lymph node and three additional lymph nodes, with a total of 4 out of 12 lymph nodes, were positive for metastases. One lymph node showed extra-nodal extension. Consequently, localized radiation to the chest wall and supraclavicular region was completed, followed by 6 cycles of adjuvant CAF (cyclophosphamide, doxorubicin, 5-flourouracil) chemotherapy. She was treated with Tamoxifen $10 \mathrm{mg}$ twice daily for 5 years following the completion of adjuvant chemoradiotherapy.

In January 2012, a surveillance CT scan of the chest revealed a $2.0 \mathrm{~cm}$ right upper lobe mass with hilar and mediastinal lymphadenopathy. Biopsy during mediastinoscopy confirmed metastatic adenocarcinoma consistent with breast carcinoma as the primary site, and displayed ER+, PR+, HER2- expressivity. Accordingly, the patient then completed nearly 3 years of anti-estrogen therapy with the aromatase inhibitor letrozole $2.5 \mathrm{mg}$ daily.

Letrozole was discontinued due to recurrence of the disease in the right knee. As treatment for this metastasis, the patient underwent radical resection of the right distal femur and reconstruction with a prosthesis. The tumor was diffusely ER+, and the patient was started on anti-estrogen therapy with another aromatase inhibitor, exemestane $25 \mathrm{mg}$ daily. 10 months later it was discontinued due to disease progression when a core biopsy of one of the inguinal lymph nodes revealed metastatic breast adenocarcinoma with ER 90\%, PR 0\%, and HER2 2+. The

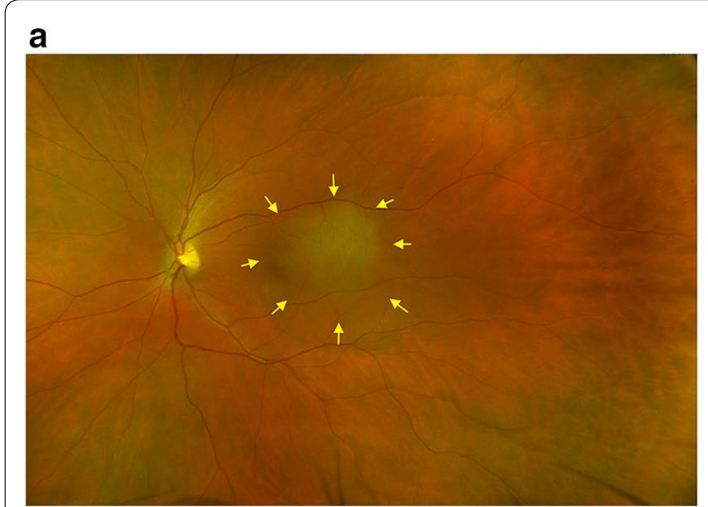

b
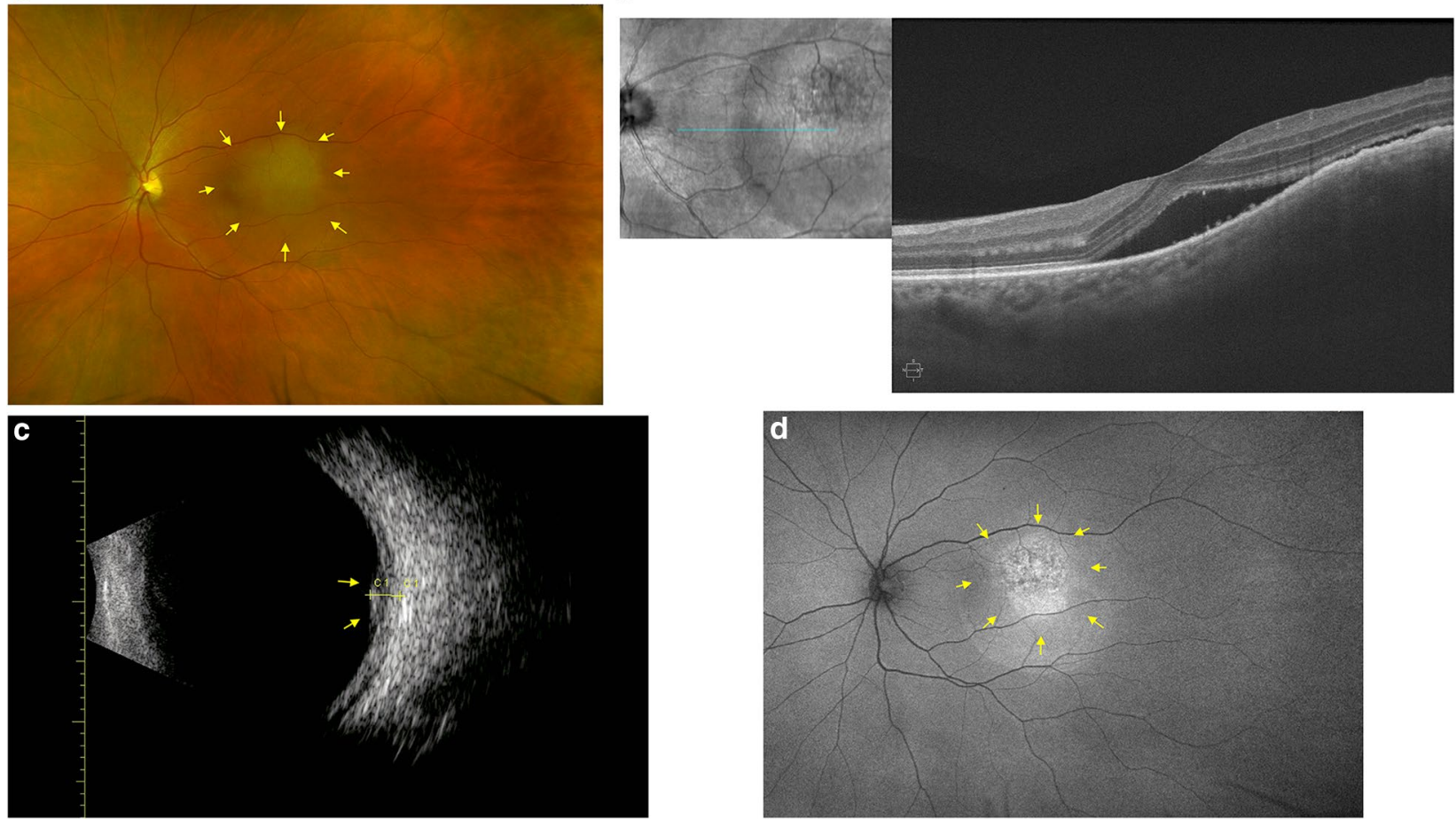

Fig. 1 Imaging studies performed in June 2017 for a 58-year-old female with choroidal metastasis from primary breast carcinoma. a The extent of the yellow-colored choroidal mass superior and temporal to the macula is marked (yellow arrows). $\mathbf{b}$ Structural OCT demonstrated subretinal fluid associated with the choroidal mass. c Ultrasound showed a $2.47 \mathrm{~mm}$ choroidal lesion (yellow arrows). $\mathbf{d}$ Fundus autofluorescence demonstrated a hyper-fluorescent lesion (yellow arrows) with surrounding subretinal fluid 
patient was then started on anti-estrogen therapy with the aromatase inhibitor fulvestrant in conjunction with the cyclin-dependent kinase inhibitor palbociclib for suspected resistance to ER endocrine therapy.

In early 2017, new lytic vertebral and left pelvic metastatic lesions were detected. The patient subsequently underwent radiation therapy to the T11-L1 region, left hip, and adjacent pelvis. She was restarted on exemestane, with the mTOR kinase inhibitor everolimus. This therapy was discontinued after 2-3 weeks due to profound weakness, nausea, and emesis. Bone scan showed a progression of osseous dissemination.

By June 2017, she had extensive osseous and lymph node dissemination. She was restarted on fulvestrant in combination with the receptor activator of nuclear factor kappa-B (RANK) ligand and osteoclast inhibitor denosumab. It was at this time that she was diagnosed with the left choroidal mass and referred for an ophthalmology consult. The patient was advised by oncology to discontinue fulvestrant due to disease progression, and was started on the mitotic inhibitor vinorelbine $25 \mathrm{mg} / \mathrm{m}^{2} \mathrm{IV}$ weekly for 2-3 weeks as tolerated.

Regarding the newly diagnosed choroidal mass, after careful consideration of radiation therapy for more definitive treatment versus photodynamic therapy (PDT) or laser to treat the subretinal fluid, a conservative approach was adopted with a 1-month follow-up to assess for possible regression of the lesion in conjunction with the initiation of vinorelbine. In July 2017, visual acuity of the left eye improved to 20/30 and funduscopic exam and color fundus photo revealed a slightly regressed choroidal mass, at $4.75 \mathrm{~mm}$, with a reduction in subretinal fluid. The OCT scan showed decreased subretinal fluid with the choroidal mass size stable at $2.47 \mathrm{~mm}$. With a possible future consideration of external beam radiotherapy (EBR) or photodynamic therapy, the patient was closely monitored for an increase in choroidal mass size and surrounding exudate in the event of a waning effect of vinorelbine.

During her last follow-up in September 2017, visual acuity of the left eye improved to 20/20. Funduscopic exam and color fundus photo revealed a regressed yellow-colored, temporally located choroidal mass (Fig. 2a). OCT demonstrated resolution of the subretinal fluid with a stable mass size of $2.47 \mathrm{~mm}$ seen on ultrasound (Fig. 2b, c). The patient continued treatment with vinorelbine and denosumab for $\mathrm{ER}+, \mathrm{PR}$-, stage IV metastatic breast carcinoma and had completed 6 cycles of vinorelbine. A

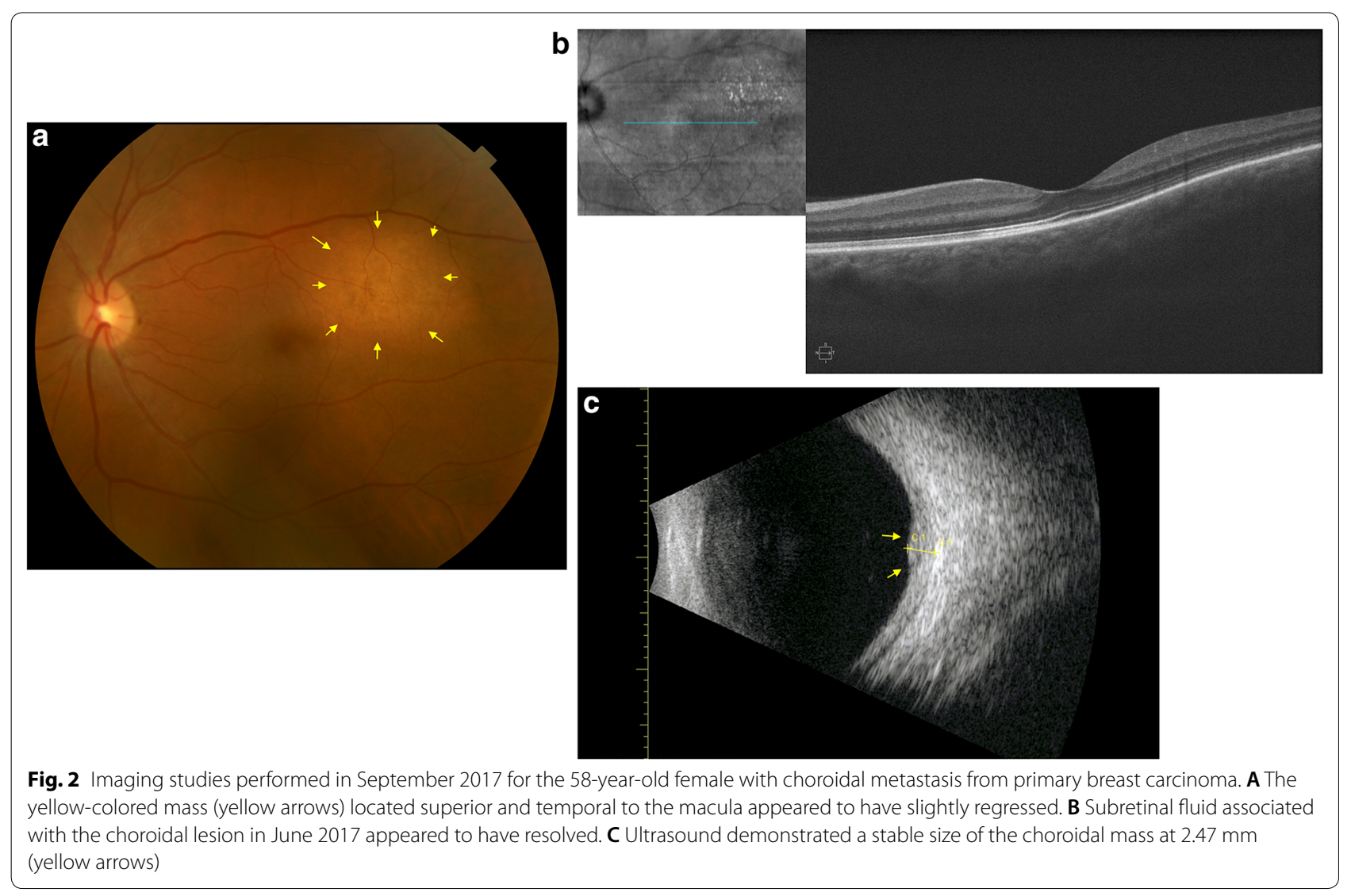


conservative approach was continued with a plan for frequent follow-up.

\section{Discussion and conclusions}

Choroidal metastatic lesions in primary breast carcinoma usually occur with systemic dissemination of the malignancy to other organs. In some patients, uveal metastatic lesions may remain asymptomatic and thus, undiscovered or discovered as a scar lesion after systemic therapy with different chemotherapeutic agents. In such cases, the systemic treatment may control the ocular disease before the ocular diagnosis.

Treatment of choroidal metastatic lesions is guided by the presence of visual symptoms. Possible treatment options for uveal metastases include systemic chemotherapy, immunotherapy, whole eye or localized radiation, indocyanine green (ICG) augmented transpupillary thermotherapy (TTT), anti-VEGF injections, and/or surgical resection. Due to lack of controlled clinical trials, there is no gold standard for their management, and therapy is driven by the treating physician's clinical judgment. Studies have demonstrated that uveal metastatic lesions may be responsive to systemic chemotherapy [912], with regression of subretinal fluid visualized on OCT as well $[13,14]$, decreasing the need for local radiation therapy [11]. Therefore, asymptomatic uveal metastatic lesions may be followed conservatively, while the patient is being treated with systemic chemotherapy.

The therapy and prognosis of primary breast carcinoma are guided by its clinicopathological characteristics, the presence or absence of hormone receptors, the expression of the HER2 oncogene, and multiparameter gene expression assays. Chemotherapy following the resection of primary breast carcinoma is recommended in all patients with high risk characteristics, such as a large tumor burden, positive lymph nodes, and/or HER2 negative status with the absence of hormone receptors. $75 \%$ of breast carcinomas are ER and PR positive [15], and these tumors demonstrate a good response to endocrine therapy. Hormone therapy with Tamoxifen in pre-menopausal women up to 5 years has been shown to reduce the risk of recurrence and decrease mortality $[16,17]$. Tamoxifen and fulvestrant are SERMs, namely estrogen antagonists that downregulate the ER protein in malignant cells. Tamoxifen is preferred in premenopausal women to preserve fertility, but comes with a risk of uterine malignancy and thromboembolism. In postmenopausal women, aromatase inhibitors are preferred to reduce the risk of recurrence, with better tolerability and without the life-threatening adverse effects of partial estrogen agonists, such as Tamoxifen [18]. Aromatase inhibitors decrease estrogen production by blocking the peripheral conversion of adrenal androgens. Tamoxifen therapy is usually recommended for 5 years; however, extended therapy for 10 years has demonstrated an additional 3\% improvement in breast cancer mortality [19]. Patients with high risk disease are usually recommended an anthracycline and taxane containing regimen, whereas those patients with low to moderate risk may be treated with a taxane (paclitaxel and docetaxel) regimen only [20]. Patients with HER2 positive disease are recommended trastuzumab and pertuzumab monoclonal antibodies in addition to chemotherapy [21].

Patients with ER+, PR+ and HER2- malignancy usually receive multiple cycles of endocrine therapy before transitioning to a single chemotherapeutic agent. Our patient developed a choroidal metastasis in $\mathrm{ER}+, \mathrm{PR}+$ and HER2 - breast carcinoma while on hormone therapy with fulvestrant. EBR therapy has a response rate of $63-83 \%$ for tumor regression [22], and was considered in this patient. However, EBR may be associated with the long-term risks of exposure keratopathy, iris neovascularization, radiation retinopathy, and optic neuropathy [23]. Arguably, the goal of therapy in metastatic disease, including uveal metastases, is mainly palliative, aiming to extend life while minimizing the adverse effects of chemotherapy and other therapeutic interventions. Therefore, in our patient, a conservative approach was adopted, accompanied by the introduction of vinorelbine, a single chemotherapeutic agent with good tolerability. With this approach, the patient has demonstrated slow, but continued resolution of the uveal metastatic lesion. To the best of our knowledge, this is the first case to report regression of choroidal metastasis with vinorelbine in an ER+, but hormone therapy resistant primary malignancy.

$\mathrm{ER}+$ metastases, such as choroidal tumors, are usually responsive to Tamoxifen or aromatase inhibitor therapy. However, some ER+ tumors are either only partially responsive to SERMs, due to ER-expression paucity, or become resistant to endocrine therapy, due to a possible mutation in kinase enzymes in the ER expression pathway, as seen in our patient. Wong et al. [11] reported complete regression of a choroidal metastasis from ER-, HER2 + breast carcinoma treated with trastuzumab and vinorelbine. Vinorelbine is a vinca alkaloid that is a semisynthetic derivative of vinblastine, with less neurotoxicity than vinblastine. Vinorelbine inhibits DNA replication mainly by preventing the assembly of microtubules in malignant cells and disrupting cell membrane function [24]. It may be a form of single-agent chemotherapy that is reasonably well-tolerated and administered weekly, with a risk of possible mild neurological, hematological, and gastrointestinal toxicity.

The regression of metastatic lesions, either partial or complete, during systemic therapy directed towards the primary malignancy is a well-known occurrence. 
However, the spontaneous regression of tumors without therapy has also been seen, although infrequent. In a series of 254 patients with choroidal metastasis from breast cancer, $18 \%$ of the patients were followed conservatively without intervention. The metastatic lesion regressed in $42 \%$ of these patients, while remaining stable in $33 \%$ of this cohort [25]. The natural regression of tumors may be attributed to programmed cell death by apoptosis, an increased presence of natural killer cells in circulation, or a modification of the tumor microenvironment that prevents tumor proliferation by inhibiting matrix metallo-proteinases and angiogenesis [26]. Additionally, p. 53 oncogene-mediated apoptosis is a pathway known to be more active in estrogen-dependent breast carcinomas [27]. Thus, spontaneous regression may have been a possible contributing factor in this case. However, since regression coincided with the initiation of vinorelbine, it was the most likely contributor to the observed regression.

Overall, we concur with earlier reports of a conservative approach for choroidal metastatic lesions in disseminated breast carcinoma while on chemotherapy [9-11, 28, 29], Additionally, we suggest the consideration of vinorelbine therapy in patients with choroidal metastasis, irrespective of hormone receptor status and responsiveness to antiestrogen therapy in disseminated breast malignancy, thereby limiting local radiation therapy to vision threatening lesions only.

\section{Abbreviations \\ ER: Estrogen receptor; SERM: Selective estrogen receptor modulator; OCT: Optical coherence tomography; HER2: Human epidermal growth factor recep- tor 2; PDT: Photodynamic therapy; ICG: Indocyanine green; TTT: Transpupillary thermotherapy.}

\section{Authors' contributions}

All authors were involved in conception and design of the study, acquisition of data, conceptualization of the manuscript and review and synthesis of the literature, analysis and interpretation of data, drafting the manuscript, and critical review and revision of the manuscript. Both authors read and approved the final manuscript.

\section{Competing interests}

Malvika Arya declares that she has no competing interests. Jay S. Duker receives research support from Carl Zeiss Meditec and OptoVue.

\section{Availability of data and materials}

Data available on request.

\section{Ethics approval and consent to participate}

The study adhered to the tenets of the Declaration of Helsinki and complied with the Health Insurance Portability and Accountability Act of 1996. Informed consent was obtaind.

\section{Financial support}

This work was supported in part by a Grant from the Lions Eye Research Fund.

\section{Publisher's Note}

Springer Nature remains neutral with regard to jurisdictional claims in published maps and institutional affiliations.

Received: 26 January 2018 Accepted: 16 April 2018

Published online: 14 May 2018

\section{References}

1. Shields CL, Shields JA, Gross NE, Schwartz GP, Lally SE. Survey of 520 eyes with uveal metastases. Ophthalmology. 1997;104:1265-76.

2. Eliassi-Rad B, Albert DM, Green WR. Frequency of ocular metastases in patients dying of cancer in eye bank populations. Br J Ophthalmol. 1996:80:125-8

3. Weiss L. Analysis of the incidence of intraocular metastasis. Br J Ophthalmol. 1993;77:149-51.

4. Redmond KJ, Wharam MD, Schachat AP. Choroidal metastases. In: Retina, vol. 3. 5th ed. Elsevier; 2012. p. 2324-9.

5. Manquez ME, Brown MM, Shields CL, Shields JA. Management of choroidal metastases from breast carcinomas using aromatase inhibitors. Curr Opin Ophthalmol. 2006;17:251-6.

6. Logani S, Gomez H, Jampol LM. Resolution of choroidal metastasis in breast cancer with high estrogen receptors. Arch Ophthalmol. 1992;110:451-2.

7. Osborne CK. Tamoxifen in the treatment of breast cancer. N Engl J Med 1998;339:1609-18.

8. Cancer Genome Atlas N. Comprehensive molecular portraits of human breast tumours. Nature. 2012;490:61-70.

9. Letson AD, Davidorf FH, Bruce RA Jr. Chemotherapy for treatment of choroidal metastases from breast carcinoma. Am J Ophthalmol. 1982;93:102-6.

10. Brinkley JR Jr. Response of a choroidal metastasis to multiple-drug chemotherapy. Cancer. 1980;45:1538-9.

11. Wong ZW, Phillips SJ, Ellis MJ. Dramatic response of choroidal metastases from breast cancer to a combination of trastuzumab and vinorelbine. Breast J. 2004;10:54-6.

12. Munzone E, Nole F, Sanna G, Goldhirsch A. Response of bilateral choroidal metastases of breast cancer to therapy with trastuzumab. Breast. 2005;14:380-3.

13. Venkatesh P, Garg S. Regression of choroidal metastasis from breast carcinoma following Letrozole therapy. Clin Exp Ophthalmol. 2007:35:492-4.

14. Singh N, Kulkarni P, Aggarwal AN, et al. Choroidal metastasis as a presenting manifestation of lung cancer: a report of 3 cases and systematic review of the literature. Medicine. 2012;91:179-94.

15. Hammond ME, Hayes DF, Dowsett M, et al. American Society of Clinical Oncology/College Of American Pathologists guideline recommendations for immunohistochemical testing of estrogen and progesterone receptors in breast cancer. J Clin Oncol. 2010;28:2784-95.

16. Early Breast Cancer Trialists' Collaborative Group. Tamoxifen for early breast cancer. Cochrane Database Syst Rev 2001;(1):CD000486.

17. Early Breast Cancer Trialists' Collaborative G, Davies C, Godwin J, et al. Relevance of breast cancer hormone receptors and other factors to the efficacy of adjuvant tamoxifen: patient-level meta-analysis of randomised trials. Lancet. 2011;378:771-84.

18. Breast International Group 1-98 Collaborative G, Thurlimann B, Keshaviah A, et al. A comparison of letrozole and tamoxifen in postmenopausal women with early breast cancer. N Engl J Med. 2005;353:2747-57.

19. Davies C, Pan H, Godwin J, et al. Long-term effects of continuing adjuvant tamoxifen to 10 years versus stopping at 5 years after diagnosis of oestrogen receptor-positive breast cancer: ATLAS, a randomised trial. Lancet. 2013:381:805-16.

20. McDonald ES, Clark AS, Tchou J, Zhang P, Freedman GM. Clinical diagnosis and management of breast cancer. J Nucl Med. 2016;57(Suppl 1):9S-16S.

21. Perez EA, Romond EH, Suman VJ, et al. Trastuzumab plus adjuvant chemotherapy for human epidermal growth factor receptor 2-positive breast cancer: planned joint analysis of overall survival from NSABP B-31 and NCCTG N9831. J Clin Oncol. 2014;32:3744-52. 
22. Kanthan GL, Jayamohan J, Yip D, Conway RM. Management of metastatic carcinoma of the uveal tract: an evidence-based analysis. Clin Exp Ophthalmol. 2007:35:553-65.

23. Rudoler $\mathrm{SB}$, Corn BW, Shields $\mathrm{CL}$, et al. External beam irradiation for choroid metastases: identification of factors predisposing to long-term sequelae. Int J Radiat Oncol Biol Phys. 1997;38:251-6.

24. Ngan VK, Bellman K, Hill BT, Wilson L, Jordan MA. Mechanism of mitotic block and inhibition of cell proliferation by the semisynthetic vinca alkaloids vinorelbine and its newer derivative vinflunine. Mol Pharmacol. 2001;60:225-32

25. Demirci H, Shields CL, Chao A, Shields JA. Uveal metastasis from breast cancer in 264 patients. Am J Ophthalmol. 2003;136:264-71.
26. Salman T. Spontaneous tumor regression. J Oncol Sci. 2016;2:1-4.

27. Wang TTY, Phang JM. Effects of estrogen on apoptosis pathways in human breast cancer cell line MCF-7. Cancer Res. 1995;55:2487-9.

28. Kosmas C, Malamos NA, Antonopoulos M. Complete regression of choroidal metastases from breast cancer after docetaxel-based systemic chemotherapy. Med Pediatr Oncol. 2000;34:229-30.

29. Shome D, Jayadev C, Gadgil D, Natarajan S, Jain V. Systemic chemotherapy and tamoxifen induced regression of choroidal metastasis from a breast carcinoma in a male. Indian J Ophthalmol. 2007:55:475-7.
Ready to submit your research? Choose BMC and benefit from:

- fast, convenient online submission

- thorough peer review by experienced researchers in your field

- rapid publication on acceptance

- support for research data, including large and complex data types

- gold Open Access which fosters wider collaboration and increased citations

- maximum visibility for your research: over $100 \mathrm{M}$ website views per year

At BMC, research is always in progress.

Learn more biomedcentral.com/submissions 